\title{
De las ondas a los 140 caracteres. El uso de Twitter por los principales programas de la radio española
}

\section{Teresa Piñeiro Otero ${ }^{1}$}

Recibido: 2014-11-10

Enviado a pares: 2014-11-11
Aprobado por pares: 2014-12-03

Aceptado: 2014-12-03

DOI: $10.5294 /$ pacla.2015.18.3.8

Para citar este artículo / To reference this article / Para citar este artigo

Piñeiro Otero, T. (Septiembre de 2015). De las ondas a los 140 caracteres. El uso de

Twitter por los principales programas de la radio española. Palabra Clave, 18(3), 815-841.

DOI: 10.5294/pacla.2015.18.3.8

\section{Resumen}

El proceso de radiomorfosis ha conllevado la ampliación del universo de la radiofonía a nuevas plataformas web como las redes sociales. Cadenas, emisoras, espacios o comunicadores radiofónicos han creado sus propios perfiles en estas plataformas, mayoritariamente en Twitter y Facebook. Estos perfiles constituyen una expansión de los contenidos de la radio convencional para una audiencia que, cada vez más, acude a Internet para ampliar la información de los medios tradicionales. Si en un primer momento la creación de perfiles sociales por las marcas radiofónicas respondía a una tendencia, en el contexto actual la apropiación de dichos canales constituye una necesidad, especialmente desde aspectos como la personalización de contenidos, la participación de los oyentes-usuarios o la consolidación de comunidades online. El presente trabajo analiza la presencia y utilización que los principales programas de la radio española dan a sus canales en Twitter. Especialmente se ha prestado atención al grado de adaptación de los espacios radiofónicos a esta plataforma atendiendo a aspectos como la frecuencia de actualización y de publicación de links, hashtags, menciones y retuits,

1 Universidade da Coruña, España. teresa.pineiro@udc.es 
así como a otras variables relacionadas con la influencia de estos perfiles en la red de microblogging.

\title{
Palabras clave
}

Radio, redes sociales, Twitter, participación, interacción, audiencia social (Fuente: Tesauro de la Unesco).

\section{From the Waves to 140 Characters. The Use of Twitter by Leading Spanish Radio Programs}

\begin{abstract}
The morphosis radio process has led the expansion of the universe of radio to new web platforms such as social networks. Chains, stations, spaces or radio journalists have created their own profiles on these platforms, mainly on Twitter and Facebook. These profiles are an expansion of the content of the conventional radio to an audience that increasingly turn to Internet to expand the information of traditional media. If at first the creation of social profiles by radio brands responding to a trend in the current ownership of these channels is a necessity, especially since aspects such as customizing content, audience participation-users or consolidation of online communities. This paper analyzes the presence and use that the main Spanish radio programs give their channels in Twitter. Particular attention has been paid to the degree of adaptation of radio programs to this platform considering aspects such as the frequency of updating and publishing links, hashtags, mentions and retweets, as well as other variables related to the influence of these profiles in the microblogging network.
\end{abstract}

\section{Keywords}

Radio, social networks, Twitter, participation, interaction, social audience (Source: Unesco Thesaurus). 


\section{Das ondas aos 140 caracteres. 0 uso do Twitter pelos principais programas da rádio espanhola}

\section{Resumo}

O processo de radiomorfose levou a ampliação do universo da radiofonia a novas plataformas web como as redes sociais. Cadeias, emissoras, espaços ou comunicadores radiofônicos criaram seus próprios perfis nessas plataformas, principalmente no Twitter e no Facebook. Esses perfis constituem uma expansão dos conteúdos da rádio convencional para uma audiência que, cada vez mais, usam a internet para ampliar a informação dos meios tradicionais. Se, em um primeiro momento, a criação de perfis sociais pelas marcas radiofônicas respondia a uma tendência, no contexto atual, a apropriação desses canais constitui uma necessidade, especialmente a partir de aspectos como a personalização de conteúdos, a participação dos ouvintes-usuários ou a consolidação de comunidades on-line. O presente trabalho analisa a presença e a utilização que os principais programas da rádio espanhola dão a seus canais no Twitter. Especialmente, prestou-se atenção ao grau de adaptação dos espaços radiofônicos a essa plataforma atendendo a aspectos como a frequência de atualização e de publicação de links, hashtags, menções e retweets, assim como a outras variáveis relacionadas com a influência desses perfis na rede de microblogging.

\section{Palavras-chave}

Rádio, redes sociais, Twitter, participação, interação, audiência social (Fonte: Tesauro da Unesco). 


\section{Introducción}

La revolución tecnológica abanderada por Internet ha propiciado la aparición de escenarios de interacción, entre la arena real y virtual, donde medios y públicos constituyen nodos de una red multimodal regida por nuevas reglas.

En el caso concreto de la radio, la transposición del medio convencional a la web no sólo ha dotado al medio de una extensión asincrónica de gran potencial (Peña Jiménez, 2010), también ha implicado la adopción de nuevas estrategias de participación. Tal como señalan Marín Montín y Romero Vejarano (2012), las cadenas de radio han percibido en estas fórmulas de participación una oportunidad para la atracción de oyentes en un contexto de gran competencia por las audiencias.

Desde las primeras iniciativas de radio en la red, los mecanismos para la participación online de los oyentes han variado sustancialmente: desde los correos electrónicos, foros, chats, encuestas, entrevistas online y blogs hasta —últimamente- las redes sociales (Martínez Costa, Moreno \& Amoedo, 2012).

El salto de la radio hertziana a la red, que Prata (2009) denominó "radiomorfosis", no ha conllevado un proceso de sustitución del medio convencional, sino que implica el enriquecimiento del modelo de transmisión lineal con las aplicaciones interactivas de Internet que ya conforman la "matriz radiofónica” (Peña Jiménez, 2012). Cada vez más las audiencias acuden a la red para ampliar y complementar la información recibida por los medios convencionales (English, Sweetser \& Ancu, 2011).

Esta oportunidad de personalizar el acceso a los contenidos, atendiendo a los propios intereses y necesidades, se debe situar en un contexto de eclosión de los dispositivos tecnológicos personales (especialmente los dispositivos móviles de altas prestaciones) que llevarán al desarrollo de un consumo individual del medio (Arango-Forero, 2013).

Como resultado de esta convergencia, los contenidos del medio se construyen en torno a la participación de los oyentes-usuarios, en su sen- 
tido más amplio, así como a la interacción entre éstos y los productores radiofónicos en el mismo plano.

Si en la radio convencional las estrategias participativas suponían un intercambio provisional de papeles entre el emisor y el receptor (Herrera, 2007), en el contexto online la rotación entre ambos roles es continua. Especialmente en el ámbito de las redes sociales, plataformas que aportan una nueva dimensión de la participación como espacios de convivencia y conectividad definidos a través de intercambios dinámicos entre sujetos, que permiten la multiedición y la retroalimentación constante (Caldevilla Domínguez, 2010).

El medio radiofónico camina hacia contenidos más interactivos, especializados y personalizados (Ala-Fossi, Lax, O'Neill, Cand \& Shaw, 2008) que, como señalan Warner, Turner y Hawthorne (2013), pretenden atraer a los usuarios de las nuevas tecnologías.

Además de los oyentes, la nueva comunidad radiofónica alcanza a los usuarios de las redes sociales, quienes también asumen el papel de productores de contenidos. Los medios cuentan con una audiencia potencial en estas plataformas cuyo valor añadido reside en la provisión de espacios de comunicación y socialización (Lara, 2008). Estas comunidades de oyentes-usuarios están formadas por personas físicamente dispersas pero conectadas e interesadas por los escenarios de la virtualidad (Arango-Forero, 2013).

En el marco de las redes sociales los oyentes se convierten en generadores de ideas, contenidos y conversación (Martínez Costa, Moreno \& Amoedo, 2012). De sus inicios como espacios lúdicos de encuentro casual, estas plataformas sociales han pasado a convertirse en comunidades cohesionadas en torno a intereses comunes en las que la interacción entre sus miembros genera una opinión pública dinámica (Fernández Hermana, 2008). Asimismo, la charla en torno a un determinado espacio radiofónico puede tener valor prescriptivo, propiciando la conversión de usuarios de las redes sociales a oyentes. 
Desde una perspectiva transmedia, la incorporación de estas plataformas al universo de la radiofonía también permite que los oyentes-usuarios se conviertan en seguidores de los conductores de sus programas de cabecera e, inclusive, les da la opción de interactuar con éstos. De este modo se da un paso más en la proximidad del medio con sus comunidad online, dado que la interacción resulta ser más fluida entre usuarios y comunicadores que entre un medio-programa y los restantes perfiles (Marta-Lazo \& García-Idiakez, 2014).

La expansión de la radio a las redes sociales y su consecuente repercusión en la personalización de contenidos y en la interacción de la comunidad de oyentes-usuarios ha dado lugar a diversas aproximaciones en los últimos años. En el ámbito concreto de la radio española, autores como Herrero Gutiérrez (2011), Peña Jiménez (2012), Herrera y Requejo (2012a, b y c), Martínez, Alguacil y Calvo (2012), Marín Montín y Romero Vejarano (2012), Ramos del Cano (2013), Díaz del Campo y Segado (2013), Videla y Piñeiro Otero (2013), Peña Jiménez y Pascual (2013), Piñeiro Otero (2014a) y Gutiérrez et al. (2014) han estudiado el salto de cadenas y programas de radio a las principales redes sociales o, en el caso de Carrera Álvarez et al. (2013) y Piñeiro-Otero (2014b), de la relación entre comunicadores radiofónicos y oyentes en Twitter.

En esta línea se inscribe el presente artículo en tanto que analiza la presencia y utilización que los programas de radio españoles dan a la plataforma de microblogging, para determinar su grado de apropiación y la relación establecida con su comunidad de oyentes-usuarios.

\section{Buenas prácticas para la gestión de perfiles radiofónicos en Twitter}

Hoy prácticamente todas las marcas radiofónicas han ampliado su presencia a las redes sociales, especialmente a Facebook y Twitter. Esta expansión no ha seguido un patrón común: por un lado destacan diversos niveles de apropiación de las redes sociales en lo que se refiere a la adaptación al lenguaje y a sus posibilidades; por otro se pueden determinar preferencias de 
una u otra plataforma en función del tipo de emisora (generalista y especializada) y su comunidad de oyentes-usuarios.

En múltiples ocasiones la creación de un perfil en redes sociales no implica la adecuación de los contenidos publicados a estas nuevas plataformas de difusión. Una cuestión que resulta destacable, dado que lo relevante no es tanto "estar" en estos gestores 2.0 como dotarlos de contenidos de calidad y fomentar la participación para conformar una verdadera comunidad de seguidores (Casacuberta \& Gutiérrez-Rubí, 2010).

Una buena gestión de redes sociales implica una actualización constante de los perfiles, la correcta identificación del medio o programa y de sus canales web, la escucha e interacción con los oyentes-usuarios, la publicación de contenidos de calidad, la difusión de archivos multimedia o de vínculos a éstos, la inclusión de llamadas a la acción para estimular la participación o la cobertura en tiempo real de determinados eventos de interés (Orihuela, 2011; Sherman, 2009; Herrera \& Requejo 2012d). En resumen, se trata de abandonar el modelo de difusión tradicional para hacer un uso eficiente de las potencialidades de dichas plataformas, como la inmediatez, la interactividad o la compartibilidad de contenidos (que favorecería una influencia más allá del perfil del medio).

En el ámbito de Twitter, algunas buenas prácticas son la creación de una bio informativa (descripción del programa, cadena, país, url de otros canales web, etc.); la creación de contenidos ad hoc para la plataforma, el seguimiento de un número relevante de tuiteros y la elaboración de listas; el uso de hipervínculos, retuits (de publicaciones de oyentes-usuarios u otros perfiles como, por ejemplo, de comunicadores), y otras prácticas como los \#FF (Follow Friday).

Frente a la difusión de información, el objetivo principal de los perfiles mediáticos en las redes — siguiendo a Eltringhan (en Herrera \& Requejo, 2012a, d) - es fomentar el community engagement. Un objetivo que, como reflejan los trabajos de Herrera y Requejo (2012a), no parece haber sido asumido por la radio española. 
La integración de estas plataformas a la radiofonía constituye una oportunidad para implicar a la audiencia, escucharla y dar respuesta a sus demandas dentro de las posibilidades del medio (Gallego, 2012). El volumen y "sentimiento" de los comentarios en torno a un programa radiofónico en redes sociales constituye un barómetro de su repercusión y del grado de compromiso de su audiencia social (Martín González, 2012).

En un contexto en el que el que la inflación de contenidos mediáticos se incrementa a la par que el déficit de atención de los oyentes-usuarios, estimular esta charla social puede generar un mayor consumo de las cadenas o programas radiofónicos (Gallego, 2013). La atracción y conservación de esta audiencia online puede ya equipararse a la offline a efectos de atracción de anunciantes e inversores (McDowell \& Dick, 2003).

\section{Metodología}

La investigación sobre la radio en Twitter en el contexto español se ha centrado en las cadenas de radio (Herrera \& Requejo 2012a, b, c; Videla \& Piñeiro, 2013) o en tipologías concretas de programas como los despertadores musicales (Martínez, Alguacil \& Calvo, 2012), prime time (Peña Jiménez \& Pascual, 2013; Gutiérrez et al., 2014) o deportivos (Marín Montín \& Romero Vejarano, 2012).

En esta línea, el presente trabajo se ha centrado en los principales programas de las cadenas de radio española con cobertura nacional en función de su índice de audiencia.

Tomando como referencia la segunda ola de 2013 del Estudio General de Medios, se estableció una muestra de partida de 51 programas de radio (generalista y especializada) con una audiencia superior a los 29 000 oyentes (una vez eliminadas duplicidades y los espacios de fórmula musical o de todo noticias). De esa selección de programas solamente el $76 \%$ (39 espacios) cuentan con un perfil propio, independiente del de la cadena de emisión. 


\section{Tabla 1}

\section{Programas de la muestra}

\begin{tabular}{|c|c|c|c|c|}
\hline Programa & Dia & Hora & Cadena & Audiencia \\
\hline Hoy por Hoy & $\mathrm{L}-\mathrm{V}$ & $6: 00-12: 30$ & Ser & 3132000 \\
\hline Herera en la Onda & L-V & $6: 00-12: 30$ & Onda Cero & 2106000 \\
\hline A Vivir que Son Dos Días & S-D & $8: 00-12: 00$ & Ser & 1980000 \\
\hline Carrusel & S-D & $15: 00-23: 00$ & Ser & 1889000 \\
\hline Del $40 \mathrm{Al} 1$ & $S$ & $10: 00-14: 00$ & $\mathrm{C} 40$ & 1875000 \\
\hline Anda ya & L-S & $6: 00-10: 00$ & $\mathrm{C} 40$ & 1692000 \\
\hline Hora 25 & L-V & $20: 00-24: 00$ & Ser & 1352000 \\
\hline Buenos Dias, Javi Nieves & $\mathrm{L}-\mathrm{V}$ & $6: 00-10: 00$ & $\mathrm{C} 100$ & 1115000 \\
\hline El Larguero & L-D & $0: 00-1: 30$ & Ser & 1100000 \\
\hline Tiempo de Juego & S-D & $16: 00-2: 00$ & Cope & 1090000 \\
\hline Te Doy Mi Palabra & S-D & $8: 00-12: 00$ & Onda Cero & 1045000 \\
\hline No Es Un Día Cualquiera & S-D & $8: 00-13: 00$ & Rne1 & 1045000 \\
\hline Dia Tal Cual & $S$ & $10: 00-14: 00$ & Dial & 1040000 \\
\hline ¡Atrévete! & L-S & $6: 00-10: 00$ & Dial & 1014000 \\
\hline La Mañana & $\mathrm{L}-\mathrm{V}$ & $6: 00-12: 00$ & Cope & 1011000 \\
\hline El Día Menos Pensado & $\mathrm{L}-\mathrm{V}$ & $6: 00-12: 00$ & Rne1 & 992000 \\
\hline La Ventana & $\mathrm{L}-\mathrm{V}$ & $16: 00-20: 00$ & Ser & 880000 \\
\hline Radio Estadio & S-D & $16: 00-1: 01$ & Onda Cero & 671000 \\
\hline Buenos Días El Pulpo & S-D & $7: 00-11: 00$ & $\mathrm{C} 100$ & 651000 \\
\hline Fin De Semana & $\mathrm{D}$ & $10: 00-14: 00$ & Cope & 641000 \\
\hline Hora 14 & L-V & $14: 00-15: 00$ & Ser & 627000 \\
\hline Julia en la Onda & L-V & $16: 00-19: 00$ & Onda Cero & 626000 \\
\hline La Brújula & $\mathrm{L}-\mathrm{V}$ & $20: 00-24: 00$ & Onda Cero & 576000 \\
\hline La Linterna & L-V & $19: 00-24: 00$ & Cope & 500000 \\
\hline Yu: No Te Pierdas Nada & $\mathrm{L}-\mathrm{V}$ & $21: 00-24: 00$ & $\mathrm{C} 40$ & 454000 \\
\hline Matinal Ser & L-S & 7:00-8:00 & Ser & 448000 \\
\hline Hablar por Hablar & $\mathrm{L}-\mathrm{V}$ & $1: 30-4: 30$ & Ser & 431000 \\
\hline El Partido de las 12 & $\mathrm{~L}-\mathrm{V}$ & $24: 00-2: 00$ & Cope & 420000 \\
\hline Milenio 3 & $S$ & $1: 30-4: 30$ & Ser & 408000 \\
\hline Los Toros & $\mathrm{D}$ & $1: 30-3: 00$ & Ser & 367000 \\
\hline Tablero Deportivo & S-D & $16: 00-24: 00$ & Rne1 & 363000 \\
\hline El Gallo Máximo & $\mathrm{L}-\mathrm{V}$ & 06:00-10:00 & Máxima & 352000 \\
\hline $\mathrm{Al}$ Primer Toque & L-V,D & $24: 00-2: 00$ & Onda Cero & 339000 \\
\hline Morning & $\mathrm{L}-\mathrm{V}$ & 06:00-10:00 & M80 & 338000 \\
\hline La Script & $S$ & $15: 00-16: 00$ & Ser & 335000 \\
\hline Diario de las 2 & $\mathrm{~L}-\mathrm{V}$ & $13: 00-15: 00$ & Rnel & 318000 \\
\hline
\end{tabular}




\begin{tabular}{|l|c|c|c|c|}
\hline \multicolumn{1}{|c|}{ Programa } & Dia & Hora & Cadena & Audiencia \\
\hline Marcador & S-D & $16: 00-0: 00$ & Radio Marca & 304000 \\
\hline Gente Viajera & S-D & $12: 00-14: 00$ & Onda Cero & 285000 \\
\hline Café Olé & L-V & $7: 00-11: 00$ & Radio Olé & 274000 \\
\hline La Tarde & L-V & $15: 00-19: 00$ & Cope & 274000 \\
\hline La Mañana Fs & S-D & $6: 00-8: 00$ & Cope & 270000 \\
\hline Classic Box & L-D & $10: 00-14: 01$ & M80 & 253000 \\
\hline Ser Aventureros & S & $6: 00-7: 00$ & Ser & 243000 \\
\hline Hot Mix & V,S & $24: 00-2: 00$ & C40 & 169000 \\
\hline Mediodía Cope & L-V & $14: 00-15: 00$ & Cope & 150000 \\
\hline La Mar de Noches & D-J & $0: 00-2: 00$ & C40 & 102000 \\
\hline La Parroquia Del Monaguillo & L-V & $2: 00-4: 00$ & Onda Cero & 81000 \\
\hline El Año del Vinilo & D & $21: 00-23: 00$ & M80 & 73000 \\
\hline El Ojo Crítico & L-V & $19: 00-20: 00$ & Rne1 & 58000 \\
\hline La Noche & L-D & $2: 00-6: 00$ & Cope & 54000 \\
\hline Enredados & S & $17: 00-18: 00$ & Rne1 & 29000 \\
\hline
\end{tabular}

Elaboración propia a partir del Estudio General de Medios. Segunda ola de 2013.

Con la finalidad de determinar la utilización que los programas de la muestra dan a Twitter, se efectuó un análisis descriptivo de carácter exploratorio de los perfiles de los programas de la radio española en Twitter a partir de una ficha de trabajo organizada en cuatro ítems: 1) comunidad de oyentes-usuarios (número de seguidores, proporción de seguidores online respecto a offline), 2) frecuencia de publicación (frente a la media de publicaciones/día se optó por la variable publicaciones/día de emisión para promover la representatividad de los programas con menor presencia en la parrilla de programación) y tipología de contenidos vehiculados, 3 ) interacción con la audiencia (retuits de otros perfiles, de los contenidos del programa por otras cuentas, porcentaje de respuesta a los usuarios) e índice de participación de los usuarios (índice sintético que resulta de la suma del feedback -retuits y marcado como favorito- divido entre los followers del perfil), y 4) influencia (índice Klout que valora actividad, temas frecuentes y su tratamiento, así como la repercusión de dichos temas entre usuarios).

Esta herramienta de análisis se ha basado en los requisitos que Lara (2008) propone para la consolidación de los medios de comunicación en redes sociales: conectividad, servicio, participación, orientación-dinamización y gestión de conocimiento. 
En su elaboración también se han tenido en cuenta las recomendaciones específicas para el uso de Twitter de autores como Orihuela (2011), Sherman (2009) y Herrera y Requejo (2012d).

Además de la información recabada de los propios perfiles (datos del 15 de octubre de 2013), también se utilizó otra tipología de herramientas de medición social como Twittonomy o Klout (herramientas que efectúan una valoración del perfil a través de diversos indicadores, desde su creación hasta la fecha de recogida de información).

\section{De los oyentes a los followers}

El estudio de los perfiles de los programas ha puesto de relevancia que, pese a la diversa extensión de las comunidades de oyentes-usuarios en Twitter, se puede determinar una cierta correlación entre la audiencia offline y los seguidores en esta plataforma.

Ocho de los diez programas de mayor audiencia offline se corresponden con los perfiles radiofónicos con más seguidores en Twitter, aunque con algunas variaciones.

Frente al predominio de audiencias EGM de los espacios matinales, son los programas deportivos los que cuentan con comunidades más extensas en la plataforma de microblogging, con una excepción: Del 40 al 1, con más de medio millón de seguidores (casi 400000 seguidores más que Tiempo de Juego, espacio situado en segundo lugar).

Resulta destacable la irrupción de La Parroquia del Monaguillo y Julia en la Onda entre los programas con mayor repercusión en la red de microblogging. Estos perfiles han logrado cohesionar extensas comunidades de oyentes-usuarios en Twitter, a través de diversas llamadas a la acción, que les han llevado a posicionarse por delante de otros programas con mayor audiencia EGM.

Especialmente relevante es el caso de La Parroquia del Monaguillo, cuyos 72065 followers dotan a esta comunidad online de una entidad similar a la offline ( 81000 radioescuchas). 


\section{Tabla 2}

\section{Principales perfiles atendiendo al número de seguidores}

\begin{tabular}{|c|c|c|c|c|c|}
\hline Programa & Dia & Hora & Tipo & Cadena & Folowers \\
\hline Del 40 Al 1 & S & $10: 00-14: 00$ & Matinal S & C40 & 550466 \\
\hline Tiempo De Juego & S-D & $16: 00-2: 00$ & Deportes Fs & Cope & 159299 \\
\hline Carrusel & S-D & $15: 00-23: 00$ & Deportes Fs & Ser & 154146 \\
\hline El Larguero & T & $24: 00-1: 30$ & Madrugada & Ser & 95002 \\
\hline Anda Ya & L-S & $06: 00-10: 00$ & Despertador & C40 & 83755 \\
\hline Herrera En La Onda & L-V & $6: 00-12: 30$ & Matinal & Onda Cero & 72081 \\
\hline La Parroquia Del Monaguillo & L-V & $2: 00-4: 00$ & Madrugada 2 & Onda Cero & 72065 \\
\hline Hora 25 & L-V & $20: 00-0: 00$ & Noche & Ser & 58620 \\
\hline Julia En La Onda & L-V & $16: 00-19: 00$ & Tarde & Onda Cero & 55885 \\
\hline Hoy Por Hoy & L-V & $6: 00-12: 30$ & Matinal & Ser & 54577 \\
\hline
\end{tabular}

Fuente: elaboración propia.

Con 88,97 followers por cada cien oyentes, el late show de Onda Cero constituye el espacio radiofónico con mayor porcentaje de seguidores online (en Twitter) por oyente offline de la muestra de estudio (tabla 3).

\section{Tabla 3}

\section{Principales perfiles con mayor porcentaje de seguidores por oyentes}

\begin{tabular}{|c|c|c|c|c|c|}
\hline Programa & Dia & Hora & Tipo & Cadena & $\begin{array}{c}\text { Followers/ } \\
\text { Oyentes }\end{array}$ \\
\hline La Parroquia Del Monaguillo & L-V & $2: 00-4: 00$ & Madrugada 2 & Onda Cero & 88,97 \\
\hline Del 40 Al 1 & S & $10: 00-14: 00$ & Matinal S & C40 & 29,36 \\
\hline Tiempo De Juego & S-D & $16: 00-2: 00$ & Deportes Fs & Cope & 14,61 \\
\hline El Gallo Máximo & L-V & $6: 00-10: 00$ & Despertador & Máxima & 12,89 \\
\hline Al Primer Toque & L-V,D & $24: 00-2: 00$ & Madrugada & Onda Cero & 11,88 \\
\hline El Ojo Crítico & L-V & $19: 00-20: 00$ & Noche & Rne1 & 9,48 \\
\hline Julia En La Onda & L-V & $16: 00-19: 00$ & Tarde & Onda Cero & 8,93 \\
\hline El Larguero & T & $24: 00-1: 30$ & Madrugada & Ser & 8,64 \\
\hline Carrusel & S-D & $15: 00-23: 00$ & Deportes Fs & Ser & 8,16 \\
\hline Yu: No Te Pierdas Nada & L-V & $21: 00-0: 00$ & Noche & C40 & 8,04 \\
\hline
\end{tabular}

Fuente: elaboración propia.

La utilización del indicador porcentaje de seguidores por oyente EGM ha permitido determinar los programas con mayor capacidad de atracción 
de usuarios en Twitter independientemente de su audiencia convencional. No obstante, pese a este factor de corrección, espacios como Del 40 al 1, Tiempo de Juego, Julia en la Onda, El Larguero o Carrusel Deportivo continúan detentando su lugar en este particular top ten.

Si se atiende a la cadena de emisión, los perfiles con mayor número de seguidores pertenecen a la programación de la radio generalista, más concretamente de Cadena Ser y Onda Cero.

\section{Actividad de los perfiles}

La creación de perfiles radiofónicos en Twitter ha ido pareja a la necesidad de dotarlos de contenidos para dar respuesta a las demandas de información de su comunidad online. Sin embargo el estudio desarrollado ha constato un volumen de publicaciones todavía limitado (figura 1).

El 57\% de los perfiles de programas de radio presentan una media de actualización inferior a los 20 tuits al día, y un $26 \%$ de las cuentas se sitúa por debajo de las diez publicaciones diarias.

\section{Figura 1 \\ Volumen de publicaciones/día de los perfiles radiofónicos}

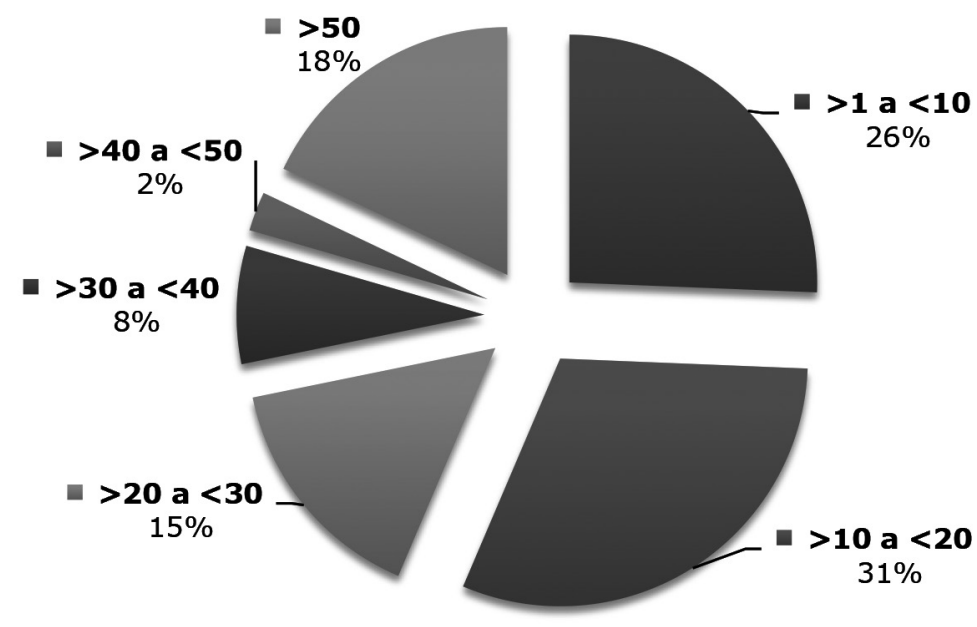

Fuente: elaboración propia. 
Resulta destacable que siete de los programas analizados presentan un volumen de actualización por encima de las 50 publicaciones/día, circunstancia que remite a una actualización continua de la plataforma.

De atender a los programas con mayor frecuencia de actualización se refrenda el peso de los programas deportivos: Tiempo de Juego (Cope Radio) y Carrusel Deportivo (Cadena Ser) superan los 100 tuits/día y otros, como El Larguero (Cadena Ser), se sitúan por encima de los 50.

El estilo sucinto de esta plataforma y la rapidez de sus publicaciones la convierten en un canal de gran valor para el ranking musical de Del 40 al 1 (Cadena 40) o destacados de entrevistas o intervenciones de La Script (Cadena Ser).

\section{Tabla 4 \\ Principales perfiles atendiendo al número de tuits/día de emisión}

\begin{tabular}{|l|c|c|c|c|c|}
\hline \multicolumn{1}{|c|}{ Programa } & Dia & Hora & Tipo & Cadena & Tuits/Día \\
\hline Tiempo De Juego & S-D & $16: 00-2: 00$ & Deportes Fs & Cope & 279,90 \\
\hline La Script & S & $15: 00-16: 00$ & & Ser & 127,96 \\
\hline Carrusel & S-D & $15: 00-23: 00$ & Deportes Fs & Ser & 104,69 \\
\hline Del 40 Al 1 & S & $10: 00-14: 00$ & Matinal S & C40 & 61,32 \\
\hline El Larguero & L-D & $0: 00-1: 30$ & Madrugada & Ser & 57,05 \\
\hline El Gallo Máximo & L-V & $6: 00-10: 00$ & Despertador & Máxima & 54,11 \\
\hline A Vivir Que Son Dos Días & S-D & $8: 00-12: 00$ & Matinal Fs & Ser & 52,64 \\
\hline Julia En La Onda & L-V & $16: 00-19: 00$ & Tarde & Onda Cero & 40,84 \\
\hline Yu: No Te Pierdas Nada & L-V & $21: 00-0: 00$ & Noche & C40 & 37,53 \\
\hline Al Primer Toque & L-V,D & $0: 00-2: 00$ & Madrugada & Onda Cero & 35,75 \\
\hline
\end{tabular}

Fuente: elaboración propia.

El volumen de publicaciones/día puede constituir un indicador de la interacción de los oyentes-usuarios. Es el caso de El Gallo Máximo (Máxima FM), cuyo perfil presenta un relevante volumen de publicaciones que son retuits de otros perfiles de la comunidad de oyentes-usuarios. 
Aunque con una menor incidencia que en el programa despertador de Máxima FM, son diversos los espacios radiofónicos que publican con frecuencia tuits de otras cuentas en sus actualizaciones de estado (figura 2).

\section{Figura 2 \\ Perfiles según el porcentaje de publicaciones que son retuits de otras cuentas}

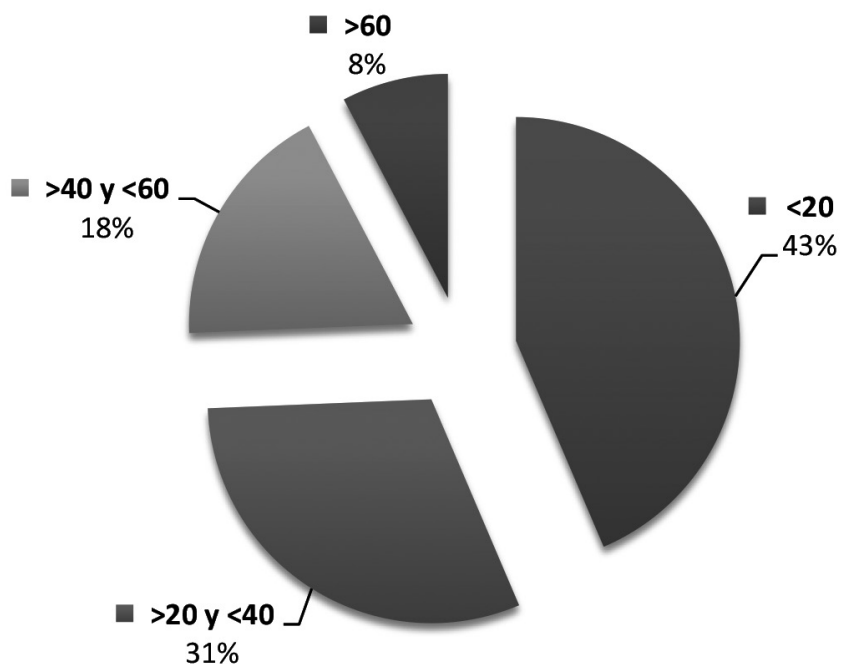

Fuente: elaboración propia.

Los retuits constituyen una fórmula bastante habitual en las publicaciones de los programas de radio en Twitter. Si bien la mayoría de las cuentas analizadas (un total de 17) presentan un volumen de publicaciones propias superior al $80 \%$, resulta destacable que en uno de cada cuatro casos (diez perfiles) dicho porcentaje se sitúe por debajo del $60 \%$.

La alimentación del perfil con contenidos de otros usuarios puede responder a una estrategia del programa (interacción, enriquecimiento del mensaje, autopromoción, etc.) o reflejar una dejadez en la gestión de dicha plataforma y la infrautilización de sus posibilidades comunicativas.

Programas como Dial Tal Cual (Cadena Dial), Ser Aventureros (Cadena Ser) o Herrera en la Onda (Onda Cero), con escasa frecuencia de 
actualización (por debajo de seis tuits/día), publican un elevado número de retuits de otras cuentas.

En el caso de Herrera en la Onda, la media de publicaciones propias es inferior a un tuit/día, habitualmente de carácter promocional (tema o invitados del día).

Por su parte, Julia en la Onda, el segundo perfil atendiendo al porcentaje de retuits, ha desarrollado en Twitter una estrategia de fomento de la participación de los usuarios; lo que lo lleva a redifundir los comentarios o respuestas de los miembros de su comunidad a sus llamadas a la acción (origen del 77\% de sus actualizaciones de perfil).

\section{Adecuación de los contenidos publicados a la plataforma}

Una de las características de Twitter como plataforma social reside en la limitación del texto de sus publicaciones a 140 caracteres. Se trata de una limitación formal en tanto que la inclusión de hipervínculos posibilita la ampliación y enriquecimiento de los contenidos.

La utilización de links, no obstante, no se ha generalizado entre los perfiles de programas radiofónicos de la muestra (figura 3). El $82 \%$ de las cuentas analizadas ( 32 perfiles) incorporan enlaces en menos del $40 \%$ de sus publicaciones, lo que implica una infrautilización de las posibilidades que brinda la plataforma.

La otra variable analizada fue la incorporación de hashtags en las actualizaciones, una utilización que facilita la conversación de un tema concreto, su posibilidad de convertirlo en un trending topic y atraer a nuevos usuarios a la conversación en una suerte de comunidad casual y temporal de oyentes-usuarios.

$\mathrm{Al}$ igual que sucedía con los links, los programas de radio no suelen utilizar los hashtags con frecuencia. El 91\% de los perfiles analizados (33 cuentas) incorpora estas etiquetas en menos del $40 \%$ de sus publicaciones.

De las ondas a los 140 caracteres. El uso de Twitter por los principales programas... - Teresa Piñeiro Otero 


\section{Figura 3 \\ Perfiles en función del porcentaje de publicaciones con links y hashtags}

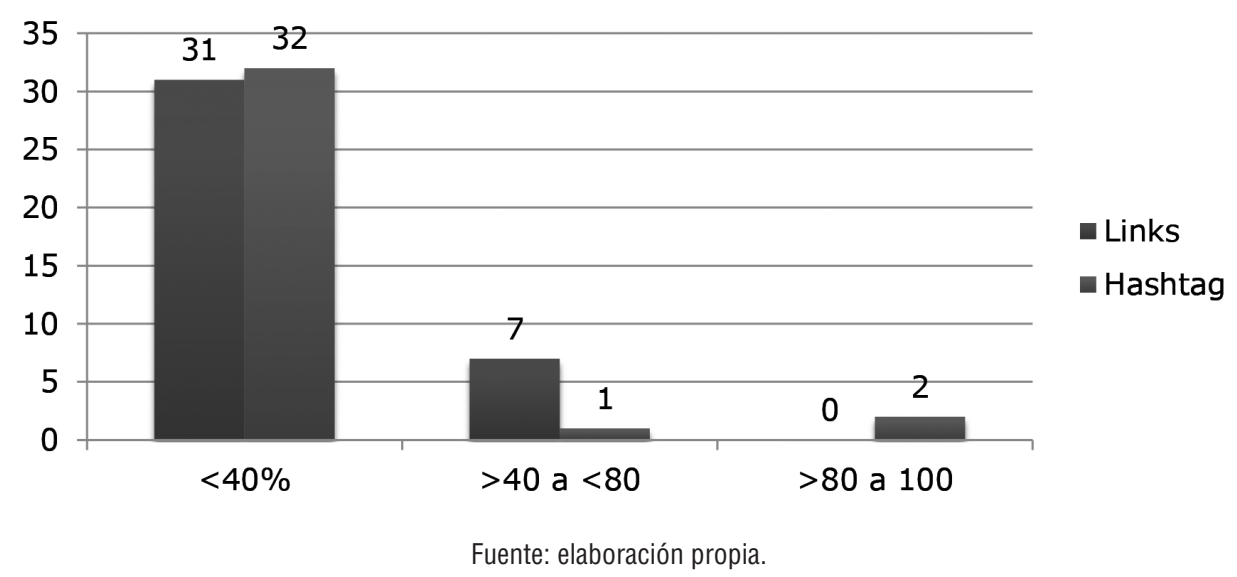

Solamente Del 40 al 1 y Radio Estadio utilizan hashtags con frecuencia para clasificar los temas y llevar la conversación más allá de su perfil, aunque no siguen una estrategia concreta para su conversión en trending topics.

De hecho, Del 40 al 1 acostumbra a utilizar \#SoyDel40al1, un hashtag global que pierde potencial viral para el algoritmo de Twitter respecto a otra tipología de hashtags puntuales o temáticos.

\section{Interacción con la audiencia}

Atendiendo al índice de interacción, el estudio desarrollado ha permitido constatar la escasa comunicación de los usuarios con los perfiles radiofónicos. Solamente en cinco casos este índice supera el $10 \%$ de las publicaciones del perfil.

Este bajo índice de interacción de los oyentes-usuarios puede vincularse a dos cuestiones: por un lado, a una concepción de la plataforma próxima a un modelo 1.0.; por otro, a una posible carencia de contenidos de interés que lleven a los receptores a asumir un papel activo.

Además del feedback "de" los usuarios, el estudio desarrollado ha prestado atención también al feedback "a" los usuarios, imprescindible en un 


\section{Figura 4 \\ Perfiles en función del índice de interacción de sus seguidores}

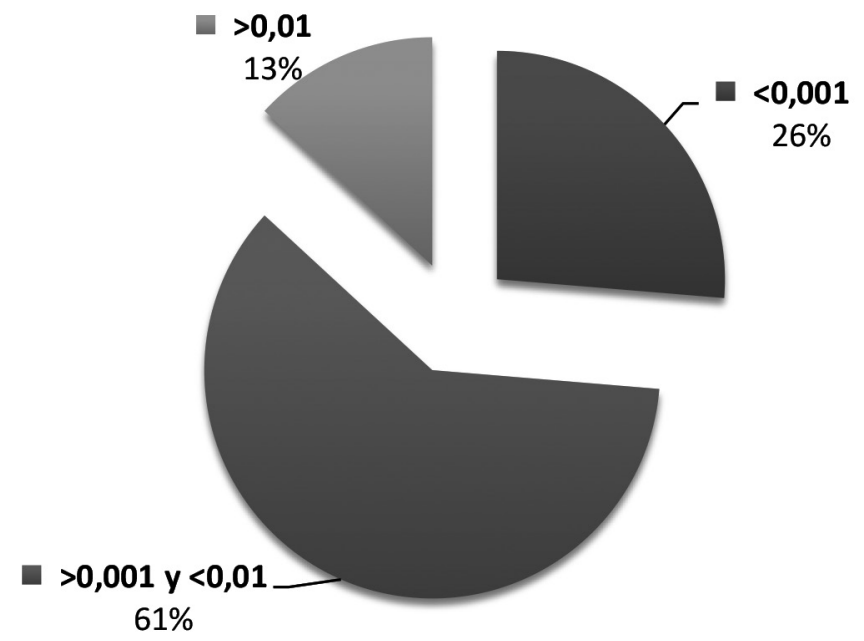

Fuente: elaboración propia.

entorno 2.0. En este caso, si bien los programas con mayor grado de adaptación a Twitter han integrado en su timeline comentarios de los oyentesusuarios o sus respuestas a las llamadas de acción, la variable retuit se ha revelado insuficiente para explicar el feedback en todos los casos (como se ha señalado en el apartado 5).

Por esta razón se ha acudido a otra variable, el porcentaje de menciones o apelaciones directas a usuarios, para determinar el feedback de los perfiles de programas radiofónicos a su comunidad de oyentes-usuarios.

Como refleja la figura 5, el análisis efectuado ha permitido destacar una mayor profusión en el uso de menciones respecto a links y hashtags. El $55 \%$ de los perfiles radiofónicos (27 cuentas) utilizan este tipo de apelaciones en un volumen importante de publicaciones (entre $40 \%$ y el $80 \%$ ), y un $15 \%$ más $(6 \%)$ las utiliza en prácticamente todas las publicaciones.

Resulta reseñable el caso de La Mañana (Cope Radio), que tiene una media de 1,37 menciones por tuit. El análisis de las publicaciones, sin em- 


\section{Figura 5 \\ Perfiles según el porcentaje de menciones que publican}

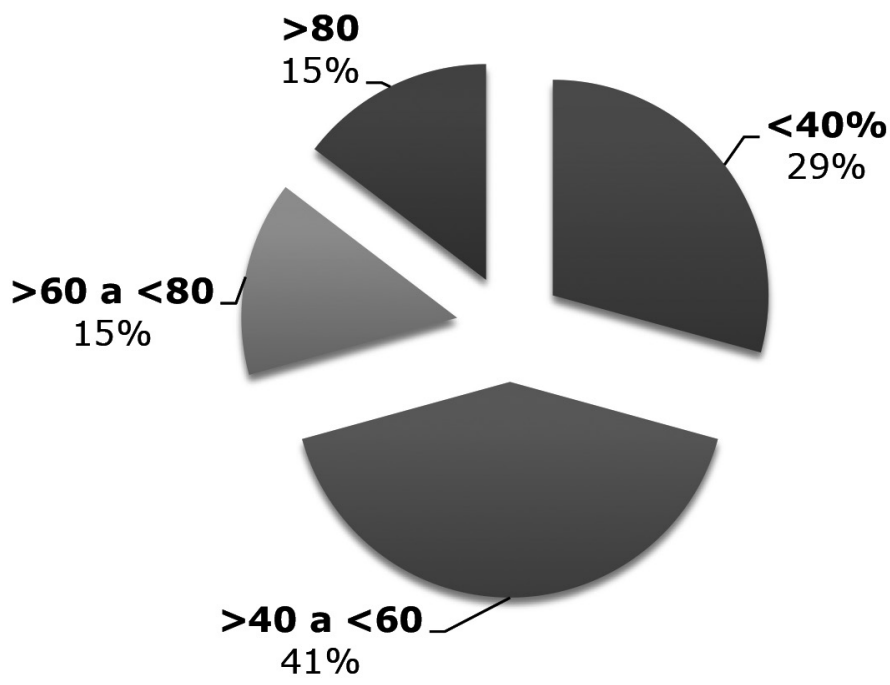

Fuente: elaboración propia.

bargo, refleja la utilización de apelaciones directas a los productores del medio o los protagonistas del día más que a los oyentes-usuarios.

\section{Influencia}

Además de datos de actividad o la interacción con la comunidad, se ha considerado la variable engagement para determinar el peso de un perfil radiofónico en Twitter.

Partiendo del índice Klout, la mitad de los perfiles de programas radiofónicos cuentan con una influencia inferior a 60, con lo que se sitúan dentro de la franja más frecuente para los perfiles en esta plataforma según señala Aguilar Granados (2013).

La otra mitad de los espacios de radio presentan un Klout superior a la media de perfiles en esta red social. Programas como Carrusel Deportivo, El Larguero y Hoy por Hoy (Cadena Ser) cuentan con una valoración en torno a los 80 puntos, que los sitúa entre los perfiles más influyentes de la tuitósfera española. 


\section{Tabla 5}

\section{Los diez perfiles con mayor influencia según su índice Klout}

\begin{tabular}{|c|c|c|c|}
\hline Programa & Dia & Hora & Tipo \\
\hline Carrusel & S-d & $15: 00-23: 00$ & Deportes fs \\
\hline El larguero & L-d & $0: 00-1: 30$ & Madrugada \\
\hline Hoy por hoy & L-v & $6: 00-12: 30$ & Matinal \\
\hline Tiempo de juego & S-d & $16: 00-2: 00$ & Deportes fs \\
\hline Al primer toque & L-v,d & $0: 00-2: 00$ & Madrugada \\
\hline Anda ya & L-s & $6: 00-10: 00$ & Despertador \\
\hline Del 40 al 1 & S & $10: 00-14: 00$ & Matinal s \\
\hline La parroquia del monaguillo & L-v & $2: 00-4: 00$ & Madrugada 2 \\
\hline La ventana & L-v & $16: 00-20: 00$ & Tarde \\
\hline Julia en la onda & L-v & $16: 00-19: 00$ & Tarde \\
\hline
\end{tabular}

Fuente: elaboración propia a partir de Klout.com

El índice Klout de los programas deportivos refrenda su preeminencia respecto a otros programas de radio en esta plataforma. Dichos espacios constituyen el $40 \%$ de los perfiles analizados con un índice superior a 65 , lo que los sitúa por delante de tuitestrellas deportivas como Cristiano Ronaldo (Klout de 40) o Iker Casillas (Klout de 61).

Asimismo resulta preciso señalar la repercusión del programa Hoy por hoy (Cadena Ser) en la plataforma de microblogging. El líder de audiencia de la radio española ha extendido su influencia a Twitter. Pese a contar con una comunidad de menor entidad, atendiendo al número de seguidores, que espacios como Herrera en la Onda (Onda Cero), el matinal de la Cadena Ser presenta un mayor engagement.

Otros espacios de gran repercusión en la red, gracias a sus constantes llamadas a la participación, son los magacines vespertinos La Ventana (Cadena Ser) y Julia en la onda (Onda Cero).

También resulta reseñable la repercusión alcanzada por La Parroquia del Monaguillo, programa que, una vez desvinculado de su horario de emisión semanal (en la franja de madrugada), ha logrado mayor influencia en Twitter que en la radio convencional.

De las ondas a los 140 caracteres. El uso de Twitter por los principales programas... - Teresa Piñeiro Otero 


\section{Conclusiones}

En el proceso de extensión de la radiofonía convencional a diversos canales 2.0 la mayoría de los programas de la radio española han ampliado su presencia web a Twitter. No obstante, la apertura de un espacio en la red de microblogging no ha ido pareja a una adaptación al lenguaje forma de la plataforma.

Tomando como referencia las recomendaciones de buenas prácticas señaladas por Orihuela (2011), Sherman (2009) y Herrera y Requejo (2012d) para la gestión de perfiles en Twitter se ha podido constatar una limitada frecuencia de actualización, al igual que del uso de hipervínculos o hashtags. Dichas tendencias reflejan una infrautilización de la agilidad e inmediatez de esta plataforma social — características que comparte con la radio-, de sus potencialidades para aportar contenidos multimedia complementarios o para generar comunidades puntuales en torno a determinados temas de conversación.

En cualquier caso, resulta preciso señalar diversos grados de apropiación de Twitter por los espacios de la radio española.

El análisis efectuado ha puesto de relevancia un predominio de los programas de cadenas generalistas, tanto si se atiende al número de seguidores como a su influencia. En lo que respecta al género, a pesar del liderazgo indiscutible de Del 40 al 1, se ha destacado el mayor peso de los programas deportivos tanto en lo que respecta al número de seguidores como a la influencia.

Si se atiende a la interacción con la audiencia resulta preciso subrayar que, en su mayoría, los programas analizados utilizan Twitter con una concepción más próxima al 1.0. que a la 2.0. Apenas se aprovechan de las posibilidades de interacción con los usuarios que ofrece la plataforma, ya se trate de la inclusión de contenidos de los seguidores en el timeline del programa (retuits), ya de la mención o apelación directa.

En el caso de los retuits, la práctica más habitual de los perfiles radiofónicos es utilizarlos para difundir los contenidos corporativos de la 
cadena, los del locutor o de otro tipo de fuentes de carácter institucional. Este uso que permite al oyente un acceso a la información relevante de la cadena se convierte en algunos perfiles en el único uso que se da a los retuits - e inclusive a las menciones-, cuestión que limita las posibilidades de feedback a la participación de los usuarios.

Al igual que subrayaron los trabajos de Herrera y Requejo relativos a la adaptación de las cadenas generalistas a Twitter, los principales programas radiofónicos parecen desconocer el lenguaje y los códigos de esta plataforma social, especialmente si se atiende a la simplicidad de sus mensajes o al escaso uso de algunos de sus recursos.

El análisis de los perfiles de programas en Twitter vuelve a manifestar la endogamia señalada por dichos autores (Herrera \& Requejo, 2012a) para los retuits, en su mayoría publicaciones de las cuentas de la cadena, de los comunicadores-colaboradores del programa e - inclusive-del grupo mediático.

En cualquier caso, además de los espacios deportivos, resulta preciso destacar la gestión que programas como Del 40 al 1 o la Parroquia del Monaguillo hacen de su perfil en la red de microblogging. Ambos programas efectúan una utilización estratégica de la plataforma tanto en su frecuencia de publicación como en su adaptación al lenguaje-forma o su capacidad de conexión e interacción con sus oyentes-usuarios. Una estrategia que se ha reflejado en su influencia dentro de esta plataforma social.

\section{Referencias}

Aguilar Granados, J. (2013). Klout, la influencia que viene. El diariofénix. com Recuperado el 5 de mayo de 2014 de http://goo.gl/OEffqN

Ala-Fossi, M., Lax, S., O'neill, B., Jauert, P. \& Shaw, H. (2008). The future of radio is still digital -but which one? Expert perspectives and future scenarios for Radio Media in 2015. Journal of Radio b Audio Media, 15(1), 4-25. Recuperado el 3 de diciembre de 2014 de http://goo.gl/DBskhq

De las ondas a los 140 caracteres. El uso de Twitter por los principales programas... - Teresa Piñeiro Otero 
Arango-Forero, G. (2013). Comunicación digital: una propuesta de análisis desde el pensamiento complejo. Palabra Clave, 16(3), 673-697. Recuperado el 3 de diciembre de 2014 de http://goo.gl/yA6iJ7

Caldevilla Domínguez, D. (2010). Las redes sociales. Tipología, uso y consumo de las redes 2.0 en la sociedad digital actual. Documentación de las Ciencias de la Información, 33, 45-68. Recuperado el 8 de junio de 2014 de http://goo.gl/11fKGQ

Carrera Álvarez, P., Herrero Curiel, E., Limón Serrano, N., Saíz de Baranda Andújar, C. \& Ocaña González, E. (2012). ¿Quién soy yo y quién eres tú? ¿Están transformando las redes sociales la imagen que los periodistas radiofónicos españoles tienen del público? Estudios sobre el Mensaje Periodístico, 18 , 223-231. Recuperado el 3 de diciembre de 2014 de http://goo.gl/y72ihc

Casacuberta, D. \& Gutiérrez Rubí, A. (2010). E-participación: de cómo las nuevas tecnologías están transformando la participación ciudadana. Razón y palabra,73. Recuperado el 12 de mayo de 2014 de http://goo.gl/znhRxv

Díaz del Campo, J. \& Segado, F. (2013). La radio en Facebook. Análisis de los perfiles de las principales emisoras y programas radiofónicos en España. Icono14, 11(2), 209-228. Recuperado el 3 de diciembre de 2014 de http://goo.gl/y72ihc

English, K., Sweetser, K. D. \& Ancu, M. (2011). YouTube-ification of political talk: An examination of persuasion appeals in viral video. American Behavioral Scientist, 55, 733-748. doi: http://doi.org/fws64n

Fernández Hermana, L. A. (2003). Investigar en tiempos de crisis ... y redes. Análisis Madri+d. Recuperado el 22 de junio de 2014 de http:// goo.gl/T0dbJj

Gallego, F. (2013). Social TV Analytics: nuevas métricas para una nueva forma de ver televisión. Index Comunicación, 3, 13-39. Recuperado el 11 de junio de 2014 de http://goo.gl/aBNQ3m 
Gallego, J. I. (2012). La audiencia en la radio: viejos roles, nuevas funciones. En Gallego, I. \& García Leiva, M. T. (Coords.), Sintonizando el futuro: Radio y producción sonora en el siglo XXI (pp. 209-228). Madrid: Instituto RTVE.

Gutiérrez, M., Martí, J. M., Ferrer, I. Monclús, B. \& Ribes, X. (2014). Los programas radiofónicos españoles de prime time en Facebook y Twitter: de inergias entre la radio convencional y las redes sociales. Revista Latina de Comunicación Social, 69, 418-434. Recuperado el 3 de diciembre de 2014 de http://goo.gl/qnRU3F

Herrera, S. (2007). La incidencia de Internet sobre la participación de los oyentes en los programas de radio. Redes.com, 4, 143-163. Recuperado el 10 de mayo de 2014 de http://goo.gl/RQqbDm

Herrera, S. \& Requejo, J. L. (2012a). Difundir información, principal uso que las emisoras de radio generalistas españolas están haciendo de Twitter. OBS Journal, 6(3), 193-227. Recuperado el 20 de junio de 2014 de http://goo.gl/F6gzxs

Herrera, S. \& Requejo, J. L. (2012b). Spanish talk Radio Stations on Twitter: Still reluctant to embrace its potential. En Oliveira, M., Portela, P. \& Santos, L. S. (Eds.), ECREA: Radio Evolution: technology, contents, audiences (pp. 49-62). Braga: Uminho. Recuperado el 3 de diciembre de 2014, de: http://goo.gl/R5qdQV

Herrera, S. \& Requejo, J. L. (2012c). La autopromoción, principal uso que las emisoras musicales españolas están haciendo de Twitter. En De Haro, M. V., Grandío, M. M. \& Hernández Pérez, M. (Coords.), Historias en red: impacto de las redes sociales en los procesos de comunicación (pp. 111-144). Murcia: Edit.um.

Herrera, S. \& Requejo, J.L. (2012d). 10 good practices for news organizations using Twitter. Journal of Applied Journalism \& Media Studies, 1(25), 79-95. Recuperado el 3 de diciembre de 2014 de http://goo.gl/ np3z3D 
Herrero Gutiérrez, F. J. (2011). Los programas deportivos de la radio española en la red social Facebook: espacio de promoción, lugar de encuentro... ¿medidor de audiencia? Área abierta, 28, 1-20. Recuperado el 12 de junio de 2014 de http:/ /goo.gl/QVHioD

Lara, T. (2008). La nueva esfera pública: los medios de comunicación como redes sociales. Telos: Cuadernos de comunicación e innovación, 76, 128-131. Recuperado el 12 de junio de 2014 de http://goo. $\mathrm{gl} / 7 \mathrm{rgLRu}$

Marín Montín, J. \& Romero Vejarano, H. J. (2012). La red social Twitter en los contenidos deportivos: nuevas perspectivas e interacciones en el actual panorama radiofónico. Actas del IV Congreso Internacional Latina de Comunicación Social. La Laguna: Universidad de la Laguna. Recuperado el 12 de julio de 2014 de http://goo.gl/KcndCv

Marta-Lazo, C. \& García-Idiakez, M. (2014). El uso profesional de la red social Twitter en la redacción del diario español El País. Palabra Clave, 17(2), 353-377. Recuperado el 3 de diciembre de 2014 de http://goo.gl/hq1nhw

Martín González, E. (2012). Televisión y redes sociales, compañeros de un largo viaje. La Comunidad Publicitaria. Recuperado el 21 de mayo de 2014 de http://goo.gl/Rt8NA5

Martínez Costa, P., Moreno, E. \& Amoedo, A. (2012). La radio generalista en la red: un nuevo modelo para la radio tradicional. Anagramas, 10(20), 165-180. Recuperado el 3 de diciembre de 2014 de http:// goo.gl/3GuRIk

Martínez, F., Alguacil Sánchez, S. M. \& Calvo Gutiérrez, E. (2011). El uso de Facebook, Twitter y otras herramientas participativas en los blogs de los programas de radio comerciales 'Anda ya', 'Atrévete' y 'Buenos días Javi y Nieves'. En Alcudia Borreguero, M. Legorburu 
Hortelano, J. M. López Delgado, L- \& Ruíz Gómez, S. (Coords.), Competidores y aliados: medios de convergencia, los nuevos retos en comunicación (pp. 349-368). Madrid: CEU Ediciones.

Mcdowell, W. \& Dick, S. J. (2003). Switching radio stations while driving: Magnitude, motivation, and measurement issues. Journal of Radio Studies, 10(1). 46-62. Recuperado el 3 de diciembre de 2014 de http://goo.gl/AHWGc8

Orihuela, J. L. (2011). Mundo Twitter. Barcelona: Alienta.

Piñeiro-Otero. T. (2014a). De la audiencia convencional a la audiencia social: Una nueva perspectiva en torno a las comunidades de los programas de radio en España. Revista Mediterránea de Comunicación, 5(1), 83-104. doi: http://doi.org/w23

Piñeiro-Otero. T. (2014b). La utilización de la bio por los programas de la radio española en Twitter. De la presentación del perfil a la radio Transmedia. Fonseca, Journal of Communication, 8, 9-34. Recuperado el 10 de julio de 2014 de http://goo.gl/5IztY5

Peña Jiménez, P. (2012). Nuevas formas de participación. Interactividad y redes sociales en la radio española. Telos. Cuadernos de Comunicación e Innovación, 92, 105-117. Recuperado el 20 de mayo de 2014 de http://goo.gl/BesiyR

Peña Jiménez, P. \& Pascual, A. (2013). Redes sociales en la radio española. Facebook, Twitter y Community Management. ZER Revista de Estudios de Comunicación, 35, 123-144. Recuperado el 10 de junio de 2014 de http://goo.gl/TU2JES

Prata, N. (2009). WEBradio: novos géneros, novas formas de interacção. Florianópolis: Editorial Insular.

Ramos del Cano, F. (2013). La cadena Ser en Facebook: nuevas estrategias de interacción con la audiencia. Fonseca Journal of Communication, 
6, 11-134. Recuperado el 12 de junio de 2014 de http://goo.gl/ HnvVLm

Sheman, A. (2009). 10 golden rules of social media. Recuperado el 12 de junio de 2014 de http://goo.gl/HBduu6

Videla Rodríguez, J.J. \& Piñeiro Otero, T. (2013). Hacia una "radio social". Interacción, proyección y repercusión de las cadenas españolas en las redes sociales. Revista ICONO14, 11(2), 83-114. doi: http:// doi.org/w24

Warner, B. R., Turner, S. \& Hawthorne, J. (2013). Limbaugh's social media nightmare: Facebook and Twitter as spaces for political action. Journal of Radio \& Audio Media, 19(2), 257-275. doi: http://doi. org/w25 